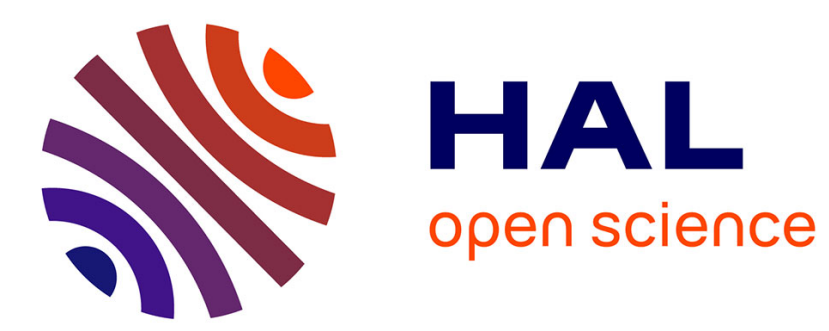

\title{
FACTEUR GYROMAGNÉTIQUE DE L'ÉTAT 12+ DES ISOTOPES DE PLOMB DE MASSE 198, 196 ET
} 194

C. Roulet, G. Albouy, G. Auger, J. Lagrange, M. Pautrat, K. Rensfelt, H. Richel, H. Sergolle, J. Vanhorenbeeck

\section{To cite this version:}

C. Roulet, G. Albouy, G. Auger, J. Lagrange, M. Pautrat, et al.. FACTEUR GYROMAGNÉTIQUE DE L'ÉTAT 12+ DES ISOTOPES DE PLOMB DE MASSE 198, 196 ET 194. Colloquium on Nuclear Surface, 1975, Dijon, France. pp.C5-95-C5-95, 10.1051/jphyscol:1975518 . jpa-00216373

\section{HAL Id: jpa-00216373 https://hal.science/jpa-00216373}

Submitted on 1 Jan 1975

HAL is a multi-disciplinary open access archive for the deposit and dissemination of scientific research documents, whether they are published or not. The documents may come from teaching and research institutions in France or abroad, or from public or private research centers.
L'archive ouverte pluridisciplinaire HAL, est destinée au dépôt et à la diffusion de documents scientifiques de niveau recherche, publiés ou non, émanant des établissements d'enseignement et de recherche français ou étrangers, des laboratoires publics ou privés. 


\title{
FACTEUR GYROMAGNÉTIQUE DE L'ÉTAT $12^{+}$ DES ISOTOPES DE PLOMB DE MASSE 198, 196 ET 194
}

\author{
C. ROUlet, G. Albouy, G. AUGer, J. M. LAGRANGe, M. PAUTRAT, \\ K. G. RENSFELT $\left({ }^{*}\right)$, H. RICHEL $\left({ }^{* *}\right)$, H. SERGOLLE et J. VANHORENBEECK $\left({ }^{* *}\right)$ \\ Institut de Physique Nucléaire, B.P. no 1, 91406 Orsay, France
}

\begin{abstract}
Résumé. - Un état isomérique $12^{+}$, interprété comme un état à deux quasi-particules $\left(v \mathrm{i} \frac{13}{2}\right)^{-2}$, a été mis en évidence dans les isotopes de plomb déficients en neutrons. 11 possède une période de quelques centaines de ns, qui se prête à la mesure du moment magnétique par la méthode des distributions angulaires différentielles perturbées (TDPAD). Ces mesures ont été réalisées, afin de confirmer la configuration de l'état et d'étudier l'effet du nombre de nucléons sur les phénomènes de polarisation du cœur.

Le tableau indique les valeurs expérimentales du facteur $g$ et de la période du niveau $12^{+}$des isotopes de masse $A=198,196,194$. Une analyse multidimensionnelle des informations $(\mathrm{E} \gamma, \mathrm{t})$ permet d'étudier simultanément ces trois noyaux, formés par la réaction naturelle $\mathrm{W}\left({ }^{16} \mathrm{O}, 4 \mathrm{n}\right) \mathrm{Pb}$. Les facteurs $g$ sont reportés sur la figure, ainsi que les valeurs obtenues par ailleurs pour l'état $12^{+} \mathrm{du}$ ${ }^{206} \mathrm{~Pb}[1],{ }^{200} \mathrm{~Pb}[2]$ et l'état $\frac{13^{+}}{2} \mathrm{du}{ }^{205} \mathrm{~Pb}[3]$.
\end{abstract}

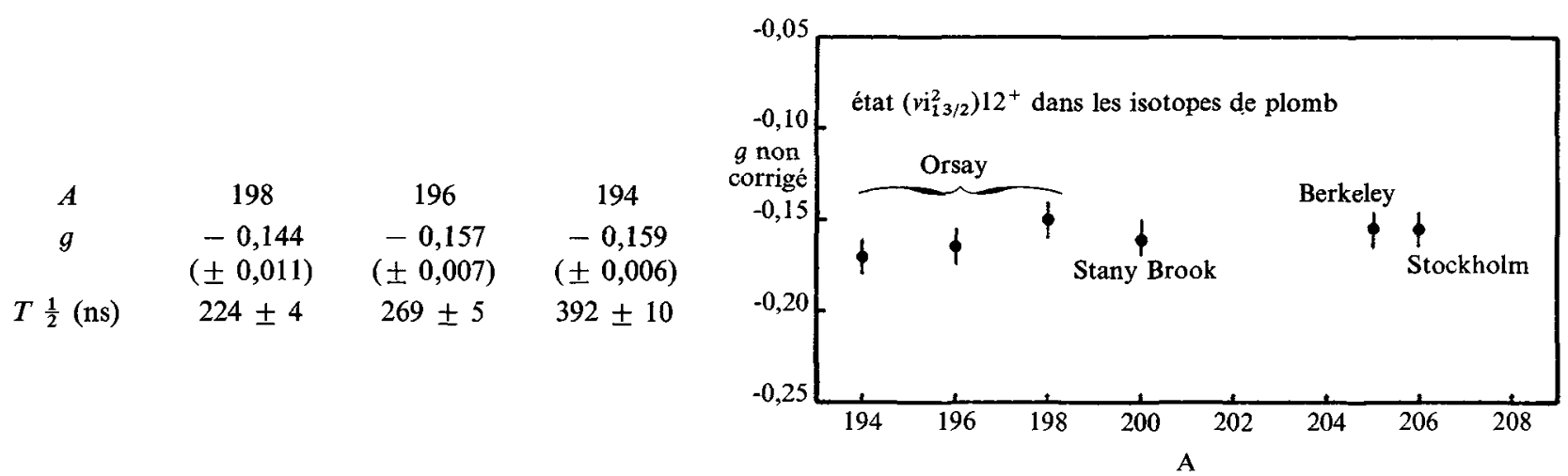

La constance de $g$ en fonction de $A$ suggère que les effets observés dans le ${ }^{206} \mathrm{~Pb}$ se retrouvent dans les isotopes plus légers, et notamment que la configuration $\left(v i \frac{13}{2}\right)^{-2}$ demeure largement dominante. L'écart notable à la valeur de Schmidt $(g=-0,294)$ peut s'expliquer par un important effet de polarisation du cœur. Un calcul, utilisant le formalisme de Arima et Horie, et une interaction résiduelle delta de surface (SDI) sont effectués pour rendre compte de ces résultats.

Abstract. - The $g$-factor of the $12^{+}$isomeric state has been determined for the three isotopes of lead with $A=194,196,198$ using the T.D.P.A.D. method. The same value obtained $(\simeq .155)$ corresponds to the $v \mathrm{i} \frac{13}{2}$ orbit $g$-factor within the experimental uncertainties. It agrees well with the expected $\left(v \mathrm{i} \frac{13}{2}\right)^{-2}$ main configuration. Additivity rule and configuration mixing due to Ml core polarization are discussed using a S.D.I. residual interaction.

\section{Bibliographie}

[1] NaKaI, K. et al., Nucl. Phys. A 189 (1972) 526.

[2] YounG, L. E. et al., Uppsala Conference (juin 1974).

[3] MaIER, K. H. et al., Nucl. Phys. A 176 (1971) 497.

Article soumis à Nuclear Physics.

(*) Research Institut for Physics, Stockholm (Suède).

$\left({ }^{* *}\right)$ Université libre de Bruxelles (Belgique). 